\title{
Analytical closed-form expressions for the structural response of helical constructions to thermal loads
}

\author{
Nikolaos Karathanasopoulos ${ }^{\mathrm{a}, *}$, Jean-Francois Ganghoffer ${ }^{\mathrm{b}}$, Konstantin O. \\ Papailiou $^{\mathrm{c}}$ \\ ${ }^{a}$ Institute for Mechanical Systems, ETH Zürich, Leonhardstrasse 21, CH-8092 Zürich, \\ Switzerland, phone: +4144633 6331; fax: +41446321145 \\ ${ }^{b}$ LEMTA - Université Lorraine - Laboratoire d'Energétique et de Mécanique, Avenue de la \\ Forêt de Haye. TSA 60604. 54504 Vandoeuvre. France \\ ${ }^{c}$ CIGRE, Malters, Switzerland
}

\begin{abstract}
In the current work, we present an analytical model for the characterization of the mechanical response of helical constructions to thermal loads. In particular, we elaborate closed-form expressions to compute the forces and moments induced by homogeneous and non-homogeneous temperature fields. We numerically verify the validity of the formulas over a wide range of geometric configurations, while we apply them to evaluate the mechanical response of two single layer cable configurations upon combined axial and thermal strains. Finally, we demarcate the range of angles for which the thermal structural response to homogeneous and non-homogeneous temperature fields considerably differs.
\end{abstract}

Keywords: Helix, Structural response, Thermal loading, Homogeneous thermal loads, Cable

\section{Introduction}

Helices are encountered in biological constructions, as for example in tendons [1], as well as in a wide range of engineering applications. In particular, they are used as the primal constituent of cables, ropes and stents $[2,3,4,5,6]$, while they are commonly found as reinforcement, or nano- and meso-structure elements $[7,8,9]$.

In order to understand and characterize their mechanical behavior, a large number of analytical models has been developed. The models have provided closed-form expressions that relate the helix material and geometric attributes

\footnotetext{
* Corresponding author

Email addresses: nkaratha@ethz.ch (Nikolaos Karathanasopoulos), jean-francois.Ganghoffer@univ-lorraine.fr (Jean-Francois Ganghoffer), konstantin@papailiou.ch (Konstantin O. Papailiou)
}

Preprint submitted to Elsevier

July 10, 2016

(C) 2016. This manuscript version is made available under the Elsevier user license http://www.elsevier.com/open-access/userlicense/1.0/ 
with the forces and moments induced by different loading patterns. More specifically, formulas characterizing the mechanical response to axisymmetric loads, in particular to axial [10], torsional [11, 12, 13] and radial loads [14] have been elaborated. What is more, closed-form expressions for the analysis of nonaxisymmetric loads, as for example bending loads have been derived [15].

The analytical models have been complemented by both laboratory experiments and numerical models. On the experimental modeling side, the mechanical response of single and multi-layer helical strands to axial loads has been measured $[16,17]$. The measurements have provided useful insights into the role of the strand's structural arrangement on the deformation profile, as well as on the forces and moments generated. On the numerical modeling side, different finite element models have been developed to simulate the helix axial and torsional loading mechanical response $[18,19,20]$, their applicability being primarily illustrated in the context of engineering helical strands [21].

In the realm of biomechanical engineering, clinical studies have been conducted to characterize the response of metallic helical stents to pressure radial loads [22]. What is more, dedicated finite element models of helical scaffolds have been constructed to provide a virtual bioengineering framework for the design of biocompatible ligament tissue engineering scaffolds [23, 24].

Dedicated numerical models have been further constructed to analyze the axial loading mechanical properties of nanoscale helical springs with different cross section profiles [25], noting their extensive applications in nanoengineering [26], as well as in microsystem technologies [27].

Along with the need to analyze and characterize the response of helical structures to mechanical loads (e.x axial, torsional or radial loads), the necessity to account for the effect of non-mechanical loads, in particular of thermal effects has become apparent in a wide range of applications. More specifically, in power transmission cable engineering, variations in the electrical load induce thermal loads, which in turn alter the structure's loading state and considerably affect its life expectancy $[28,29]$. Accordingly, in composite structure's engineering, in order to appropriately design helical bar reinforcements, the range of allowable temperature variations needs to be determined, for which analytical effective rod models and numerical models have been proposed [7]. Effective layered cylinder models have been respectively developed, to retrieve the thermal mechanical properties of carbon nanotube arrays [8]. Finally, the effect of thermal loads on the structural response of suspended cables has been elaborated, making use of the catenary equation [6].

While a large number of analytical and numerical models has been developed to characterize the structural response of helical constructions to mechanical loads, the effect of non-mechanical loads, in particular of thermal loads, has been captured by means of either effective analytical models, or numerical models. However, no analytical expression has been so far provided to explicitly account for the role of the helix geometric and material properties, as well as for the thermal loading input type.

In the current work, we derive closed-form expressions that characterize the structural response of helical constructions to homogeneous and non-homogeneous 
thermal loads. In Section 2, we describe the helix thermal loading kinematics, upon which, we formulate the structure's constitutive and equilibrium equations. By that means, we correlate the helix material and geometric attributes with the forces and moments developed, providing in Section 3.1 analytical thermal loading stiffness expressions. In Section 3.2, we employ the formulas to assess the effect of different thermal loads on the loading state of single layer, axially strained cables. Finally, we discuss on the magnitude of the forces and moments created in conjunction to the thermal field applied and conclude with a discussion of the main results in Section 4. 


\section{Mechanical model formulation}

\subsection{Helix geometry}

We describe the helix geometry as a helical fiber. The helix centerline is characterized by the position vector $\mathbf{R}(\varphi)$, defined in a Cartesian basis $\mathbf{e}_{x}, \mathbf{e}_{y}, \mathbf{e}_{z}$ as follows [30]:

$$
\mathbf{R}(\varphi)=\left\{\begin{array}{c}
a \cos \varphi \\
a \sin \varphi \\
b \varphi
\end{array}\right\}, \quad \varphi=\frac{\ell}{\gamma}, \quad \gamma=\sqrt{a^{2}+b^{2}} \quad b=a \tan \theta
$$

In Eq. 1, parameter $a$ represents the helix radius, while $b$ stands for the helix rise along the central Cartesian axis $z$ and $\gamma$ for the helix Curvilinear length per unit angular evolution $\varphi$. We schematically depict the afore-introduced helix geometric parameters in Fig. 1:

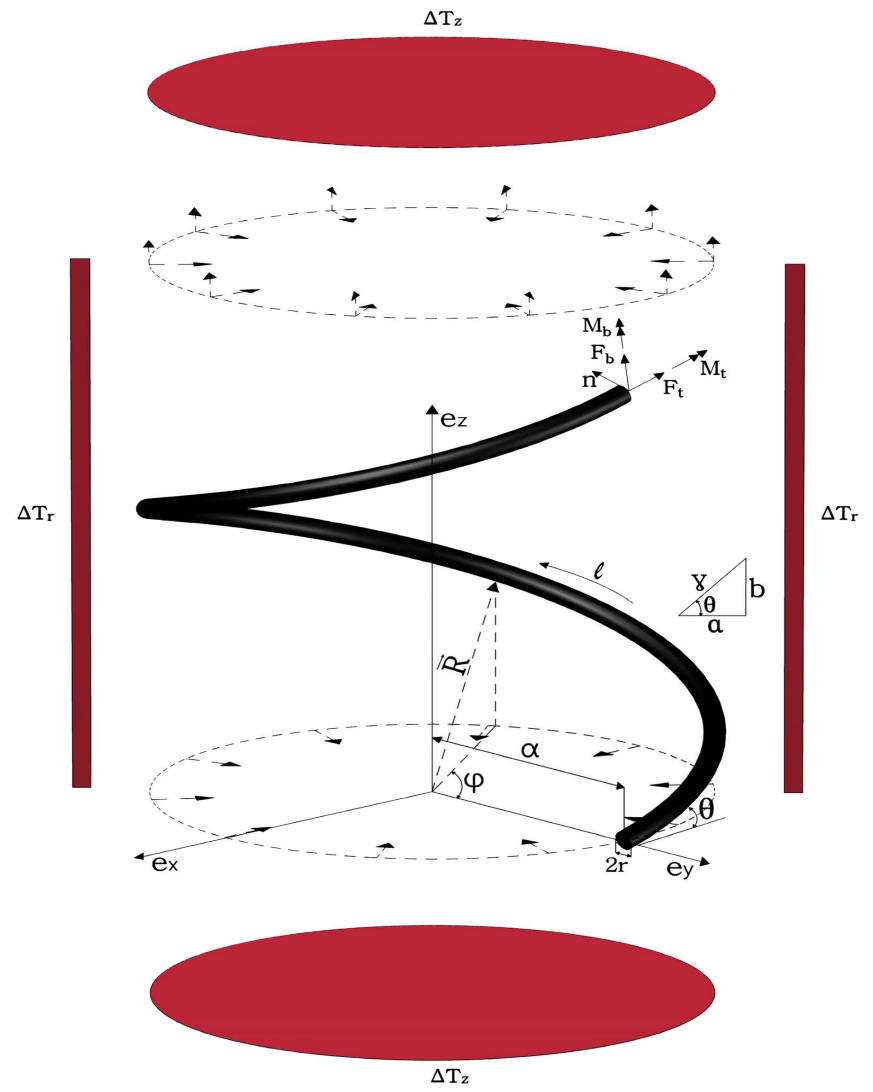

Figure 1: Helix geometry 
The parameter $b$ is related to the helix radius $a$ through the tangent of the angle $\theta$ (Eq. 1), while the helix cross section radius is denoted with $r$ (Fig. 1). The Serret-Frenet Curvilinear $\mathbf{n}, \mathbf{b}, \mathbf{t}$ basis is thereupon given as [30]:

$$
\mathbf{n}=\left[\begin{array}{c}
-\cos \varphi \\
-\sin \varphi \\
0
\end{array}\right], \quad \mathbf{b}=\frac{1}{\gamma}\left[\begin{array}{c}
b \sin \varphi \\
-b \cos \varphi \\
a
\end{array}\right], \quad \mathbf{t}=\frac{1}{\gamma}\left[\begin{array}{c}
-a \sin \varphi \\
a \cos \varphi \\
b
\end{array}\right]
$$

The helix curvature and tortuosity named respectively as $k$ and $\tau$ are defined by means of the helix radius $a$ and helix angle $\theta$ as follows [30]:

$$
k=\frac{\cos ^{2} \theta}{a}, \quad \tau=\frac{\sin \theta \cos \theta}{a}
$$

Finally, the Curvilinear helix length $\ell$ of a non-unit angular evolution $\varphi$ and its projection on the Cartesian axis $z$, named $h$, are related to the helix radius $a$ and helix angle $\theta$ by the following geometric relations [11]:

$$
h=\ell \sin \theta, \quad a \varphi=\ell \cos \theta
$$

\subsection{Helix structural response definition}

We describe the helix structural response by means of three Quantities of Interest $(Q o I)$, namely the axial force $F_{z}$ and moment $M_{z}$ created along the Cartesian axis $z$ and the radial force $F_{r}$. To compute the effective structural response $\left[\begin{array}{lll}F_{z} & M_{z} & F_{r}\end{array}\right]^{T}$, we distinguish between the mechanical loading contributions (e.g. axial, torsional or radial strains) $\left[F_{z} M_{z} F_{r}\right]_{m}^{T}$ and the non-mechanical loading contributions $\left[\begin{array}{lll}F_{z} & M_{z} & F_{r}\end{array}\right]^{T}{ }_{t h}$ induced by thermal strains:

$$
\left[\begin{array}{c}
F_{z} \\
M_{z} \\
F_{r}
\end{array}\right]=\left[\begin{array}{c}
F_{z} \\
M_{z} \\
F_{r}
\end{array}\right]_{m}-\left[\begin{array}{c}
F_{z} \\
M_{z} \\
F_{r}
\end{array}\right]_{t h}
$$

In the linear superposition of Eq. 5, the thermal part contribution $(t h)$ is subtracted from the mechanical part $(m)$, as positive signed thermal and positive signed mechanical strains induce opposite orientation force and moment resultants [31].

The structural response to mechanical strains is given in Appendix B. We hereby derive the helix thermal loading closed-form stiffness expressions, relating the thermally induced force and moment resultants of Eq. 5 to the applied thermal strains. To that extent, we express the thermal part $\left[F_{z} M_{z} F_{r}\right]^{T}{ }_{t h}$ of Eq. 5 as the multiple of a thermal loading stiffness matrix $\boldsymbol{\kappa}_{t h}$ with the thermal strain vector $\boldsymbol{\epsilon}^{t h}=\left[\epsilon_{x}^{t h}, \epsilon_{y}^{t h}, \epsilon_{z}^{t h}\right]=\left[\alpha_{t h} \Delta T_{x}, \alpha_{t h} \Delta T_{y}, \alpha_{t h} \Delta T_{z}\right]$, where $\alpha_{t h}$ stands for the linear thermal expansion coefficient (considering an isotropic thermal behavior with equal expansion coefficients) and $\Delta T_{x}, \Delta T_{y}, \Delta T_{z}$ for the temperature changes along the Cartesian $x, y$ and $z$ axis. The thermal $Q o I$ in Eq. 5 are thereupon expressed as: 


$$
\left[\begin{array}{c}
F_{z} \\
M_{z} \\
F_{r}
\end{array}\right]_{t h}=\left[\begin{array}{ccc}
\kappa_{x}^{F_{z}} & \kappa_{y}^{F_{z}} & \kappa_{z}^{F_{z}} \\
\kappa_{x}^{M_{z}} & \kappa_{y}^{M_{z}} & \kappa_{z}^{M_{z}} \\
\kappa_{x}^{F_{r}} & \kappa_{y}^{F_{r}} & \kappa_{z}^{F_{r}}
\end{array}\right]\left[\begin{array}{c}
\epsilon_{x}^{t h} \\
\epsilon_{y}^{t h} \\
\epsilon_{z}^{t h}
\end{array}\right]=\left[\begin{array}{cc}
\kappa_{r}^{F_{z}} & \kappa_{z}^{F_{z}} \\
\kappa_{r}^{M_{z}} & \kappa_{z}^{M_{z}} \\
\kappa_{r}^{F_{r}} & \kappa_{z}^{F_{r}}
\end{array}\right]\left[\begin{array}{c}
\epsilon_{r}^{t h} \\
\epsilon_{z}^{t h}
\end{array}\right]
$$

where in the rightmost equality of Eq. 6, we have considered the $z$ axis, axisymmetric thermal loading problem (Fig. 1), in which, equal thermal changes apply along the $x$ and $y$ axis $\left(\Delta T_{x}=\Delta T_{y}=\Delta T_{r}\right)$.

We elaborate a condensed form of Eq. 6 , by combining the stiffness coefficients of each $Q o I$ in a single term characterizing the non-homogeneous $(n h)$ radial-axial thermal loading case. To that scope, we express the radially applied thermal strain as a linear multiple $\left(c_{t h}\right)$ of the axial one, as follows:

$$
\epsilon_{r}^{t h}=c_{t h} \epsilon_{z}^{t h} \Rightarrow \Delta T_{r}=c_{t h} \Delta T_{z}
$$

After substituting Eq. 7 in Eq. 6, we obtain:

$$
\left[\begin{array}{c}
F_{z} \\
M_{z} \\
F_{r}
\end{array}\right]_{t h}=\left[\begin{array}{c}
c_{t h} \kappa_{r}^{F_{z}}+\kappa_{z}^{F_{z}} \\
c_{t h} \kappa_{r}^{M_{z}}+\kappa_{z}^{M_{z}} \\
c_{t h} \kappa_{r}^{F_{r}}+\kappa_{z}^{F_{r}}
\end{array}\right] \epsilon_{z}^{t h}=\left[\begin{array}{c}
\kappa_{\epsilon_{t h}}^{F_{z} n h} \\
\kappa_{\epsilon_{t h}}^{M_{z} n h} \\
\kappa_{\epsilon_{t h}}^{F_{r} n h}
\end{array}\right] \epsilon_{z}^{t h}
$$

Eq. 8 directly simplifies to the homogeneous thermal loading case $(h)$, by setting the coefficient $c_{t h}$ equal to unity, in which case $\Delta T_{r}=\Delta T_{z}=\Delta T$.

\subsection{Thermal loading kinematic relations}

We relate the applied thermal strains $\epsilon_{r}^{t h}$ and $\epsilon_{z}^{t h}$ to the helix geometry, by correlating the loading input with the helix deformation modes. The helix radial strain component $\epsilon_{r}^{t h}$ is defined as the ratio of the variation of the helix radius $\delta a$ with the undeformed helix radius $a$, while the axial strain component $\epsilon_{z}^{t h}$ is elaborated as the ratio of the variation of the $z$ axis projected helix length $\delta h$ with the undeformed helix projected length $h$ :

$$
\epsilon_{r}^{t h}=\frac{\delta a}{a} \Rightarrow \delta a=\epsilon_{r}^{t h} a, \quad \epsilon_{z}^{t h}=\frac{\delta h}{h} \Rightarrow \delta h=\epsilon_{z}^{t h} h
$$

We correlate the applied thermal strains of Eq. 9 with the helix geometric attributes, by computing the variation of the geometric quantities of Eq. 4, as follows:

$$
\begin{aligned}
\delta h & =\delta \ell \sin \theta+\ell \cos \theta \delta \theta \\
\delta(a \varphi) & =\delta a \varphi=\delta \ell \cos \theta-\ell \sin \theta \delta \theta
\end{aligned}
$$

In the second equality of Eq. 10, we note that the central angle variation $\delta \varphi$ is zero, as no torsional deformation is induced by the thermal strain field 
that is completely defined by the radial and axial thermal strain components of Eq. 9. By substituting the thermal strain definitions of Eq. 9 in Eq. 10 and by multiplying by $\cdot \cos \theta$ and $\cdot \sin \theta$ the upper and lower equality respectively, we retrieve:

$$
\begin{aligned}
& \epsilon_{z}^{t h} h \cos \theta=\delta \ell \sin \theta \cos \theta+\ell \cos ^{2} \theta \delta \theta \\
& \epsilon_{r}^{t h} a \varphi \sin \theta=\delta \ell \sin \theta \cos \theta-\ell \sin ^{2} \theta \delta \theta
\end{aligned}
$$

We proceed by subtracting the lower equality of Eq. 11 from the upper, to retrieve the angular variation $\delta \theta$ as a function of geometric terms and of the thermal straining applied to the helical body:

$$
\epsilon_{z}^{t h} h \cos \theta-\epsilon_{r}^{t h} a \varphi \sin \theta=\ell \delta \theta
$$

We further simplify Eq. 12, making use of the geometric definitions of Eq. 4, upon which we obtain:

$$
\delta \theta=\left(\epsilon_{z}^{t h}-\epsilon_{r}^{t h}\right) \sin \theta \cos \theta
$$

Eq. 13 suggests that for the case of homogeneous thermal loadings $\left(\Delta T_{r}=\right.$ $\left.\Delta T_{z}\right)$, the angular variation of Eq. 13 vanishes $(\delta \theta=0)$.

We use a total of three internal strain components to define the helix constitutive equations, as elaborated in [32] and [13]. In particular, we compute the internal normal, torsional and flexural strains that develop along the helix tangential $t$ and binormal axis $b$ (Fig. 1).

In order to compute the normal strain $\epsilon_{\ell}=\delta \ell / \ell$, we divide the first equality of Eq. 11 by $\ell$ and replace the angular variation expression $\delta \theta$ of Eq. 13, upon which, after simplifications, we obtain:

$$
\epsilon_{\ell}=\frac{\delta \ell}{\ell}=\epsilon_{z}^{t h} \sin ^{2} \theta+\epsilon_{r}^{t h} \cos ^{2} \theta
$$

We note that the helix normal thermal strain of Eq. 14 simplifies for the case of homogeneous thermal loading $\left(\epsilon_{z}^{t h}=\epsilon_{r}^{t h}\right)$ to the equivalent rod thermal normal strain expression $\epsilon_{\ell}=\alpha_{t h} \Delta T$. We compute the flexural and torsional strains denoted accordingly by $\omega_{2}$ and $\omega_{3}$, by taking into account both the initial values and variations of the helix curvature $\kappa$ and tortuosity $\tau$ provided in Eq. 3 [13]:

$$
\begin{aligned}
& \omega_{2}=\delta k+k \epsilon_{\ell}=-\frac{\sin ^{2} \theta \cos ^{2} \theta}{a} \epsilon_{z}^{t h}+\frac{\cos ^{2} \theta\left(1+\sin ^{2} \theta\right)}{a} \epsilon_{r}^{t h} \\
& \omega_{3}=\delta \tau+\tau \epsilon_{\ell}=-\frac{\sin \theta \cos ^{3} \theta}{a} \epsilon_{z}^{t h}+\frac{\sin ^{3} \theta \cos \theta}{a} \epsilon_{r}^{t h}
\end{aligned}
$$

where for the computation of Eq. 15, the rigid body motion variation parts pertaining to the helix radius change $\delta a$ have been excluded, as they do not contribute to the mechanical power created by angular rotations [32]. We further observe that in the case of homogeneous thermal loading, even though the curvature and tortuosity variations $\delta \kappa$ and $\delta \tau$ vanish $(\delta \theta=0)$, the flexural and torsional strains $\omega_{2}$ and $\omega_{3}$ remain non-zero, as of the non-zero thermal normal strain $\epsilon_{\ell}$ contribution parts $\left(k \epsilon_{\ell}\right.$ and $\tau \epsilon_{\ell}$ respectively). 


\subsection{Constitutive and equilibrium equations}

The normal, flexural and torsional strains of Eqs. 14 and 15 induce forces and moments along the local helix Serret-Frenet frame (Eq. 2), in particular along the local tangential $\mathbf{t}$ and binormal $\mathbf{b}$ base vectors. Each strain component activates the helix cross section stiffness to which it pertains, providing the following set of constitutive equations:

$$
\begin{aligned}
F_{t}^{t h} & =E A\left(\epsilon_{z}^{t h} \sin ^{2} \theta+\epsilon_{r}^{t h} \cos ^{2} \theta\right) \\
M_{b}^{t h} & =E I\left(-\frac{\sin ^{2} \theta \cos ^{2} \theta}{a} \epsilon_{z}^{t h}+\frac{\cos ^{2} \theta\left(1+\sin ^{2} \theta\right)}{a} \epsilon_{r}^{t h}\right) \\
M_{t}^{t h} & =G J\left(-\frac{\sin \theta \cos ^{3} \theta}{a} \epsilon_{z}^{t h}+\frac{\sin ^{3} \theta \cos \theta}{a} \epsilon_{r}^{t h}\right)
\end{aligned}
$$

where in Eq. 16, EA, GJ and $E I$ stand for the helix cross section axial, torsional and bending stiffness respectively. $E$ and $G$ stand accordingly for the helix linear elastic and shear material modulus parameters $(E=G /(2 \cdot(1+\nu))$, while $\nu$ is the Poisson ratio. The cross section area $A$, polar moment of inertia $J$ and bending moment of inertia $I$ are computed using the radius $r$ of the helix (Fig. 1 ), as $A=\pi r^{2}, J=\pi r^{4} / 2$ and $I=\pi r^{4} / 4$ accordingly. We note that we have used no separate expression for the binormal shear force $F_{b}^{t h}$ in Eq. 16, following the consideration that a zero moment develops around the helix normal SerretFrenet axis $\mathbf{n}$ [13]. Hereupon, the binormal force is given as a function of the local moments developed as follows:

$$
F_{b}^{t h}=\left(M_{t}^{t h} \sin \theta-M_{b}^{t h} \sin \theta\right) \frac{\cos \theta}{a}
$$

Out of the internal thermal forces $F_{t}^{t h}, F_{b}^{t h}$ and moments $M_{t}^{t h}, M_{b}^{t h}$ of Eqs. 16 and 17, we use equilibrium to retrieve the non-mechanical thermal

QoI $\left[\begin{array}{lll}F_{z} & M_{z} & F_{r}\end{array}\right]_{t h}^{T}$ of Eq. 5. To compute the radial force $F_{r}$, we make use of the moment $M_{z}$ conjugate circumferential force $F_{c}$ defined as $F_{c}=M_{z} / a$, its derivation elaborated in [14]:

$$
\left[\begin{array}{c}
F_{z} \\
M_{z} \\
F_{r}
\end{array}\right]_{t h}=\left[\begin{array}{l}
F_{t}^{t h} \sin \theta+F_{b}^{t h} \cos \theta \\
M_{t}^{t h} \sin \theta+M_{b}^{t h} \cos \theta+a\left(F_{t}^{t h} \cos \theta-F_{b}^{t h} \sin \theta\right) \\
\frac{a}{b} F_{c}^{t h}
\end{array}\right]
$$

By substituting the constitutive expressions of Eq. 16 and 17 in the equilibrium expressions of Eq. 18, we obtain, after simplifications, the following set of 
equations:

$$
\begin{aligned}
F_{z}^{t h}= & E A\left(s^{3} \epsilon_{z}^{t h}+c^{2} s \epsilon_{r}^{t h}\right)+\frac{E I}{a^{2}}\left(s^{3} c^{4} \epsilon_{z}^{t h}-s c^{4}\left(1+s^{2}\right) \epsilon_{r}^{t h}\right) \\
& +\frac{G J}{a^{2}}\left(s c^{6} \epsilon_{z}^{t h}+s^{3} c^{4} \epsilon_{r}^{t h}\right) \\
M_{z}^{t h}= & E A a\left(c s^{2} \epsilon_{z}^{t h}+c^{3} \epsilon_{r}^{t h}\right)-\frac{E I}{a}\left(s^{2} c^{3}\left(1+s^{2}\right) \epsilon_{z}^{t h}-c^{3}\left(1+s^{2}\right)^{2} \epsilon_{r}^{t h}\right) \\
& +\frac{G J}{a}\left(s^{4} c^{3} \epsilon_{z}^{t h}+s^{6} c \epsilon_{r}^{t h}\right) \\
F_{r}^{t h}= & E A\left(c^{2} s \epsilon_{z}^{t h}+\frac{c^{4}}{s} \epsilon_{r}^{t h}\right)-\frac{E I}{a^{2}}\left(s c^{4}\left(1+s^{2}\right) \epsilon_{z}^{t h}-\frac{c^{4}}{s}\left(1+s^{2}\right)^{2} \epsilon_{r}^{t h}\right) \\
& +\frac{G J}{a^{2}}\left(s^{3} c^{4} \epsilon_{z}^{t h}+s^{5} c^{2} \epsilon_{r}^{t h}\right)
\end{aligned}
$$

where the abbreviations $c$ and $s$ have been employed to denote the trigonometric factors $\cos \theta$ and $\sin \theta$. In Section 3.1, we make use of Eqs. 7 and 8 to retrieve explicit forms for the non-homogeneous and homogeneous thermal loading stiffness.

\section{Results}

\subsection{Thermal stiffness analytical formulas}

By factoring out of Eq. 19 the axial thermal strain $\epsilon_{z}^{t h}$ and by expressing the radial thermal strain $\epsilon_{r}^{t h}$ as a function of the axial, according to Eq. 7, the following closed-form expressions are obtained for the helix non-homogeneous $(n h)$ thermal loading stiffness coefficients defined in Eq. 8:

$$
\begin{aligned}
\kappa_{\epsilon_{t h}}^{F_{z} n h} & =c_{t h} \kappa_{r}^{F_{z}}+\kappa_{z}^{F_{z}}=E A s\left[c_{t h}+s^{2}\left(1-c_{t h}\right)\right] \\
& +\frac{E I}{a^{2}} s c^{4}\left[s^{2}\left(1-c_{t h}\right)-c_{t h}\right]+\frac{G J}{a^{2}} s c^{4}\left[1+s^{2}\left(c_{t h}-1\right)\right] \\
\kappa_{\epsilon_{t h}}^{M_{z} n h} & =c_{t h} \kappa_{r}^{M_{z}}+\kappa_{z}^{M_{z}}=E A a c\left[c_{t h}+s^{2}\left(1-c_{t h}\right)\right] \\
& +\frac{E I}{a} c^{3}\left(1+s^{2}\right)\left[c_{t h}+s^{2}\left(c_{t h}-1\right)\right]+\frac{G J}{a} s^{4} c\left[1+s^{2}\left(c_{t h}-1\right)\right] \\
\kappa_{\epsilon_{t h}}^{F_{r} n h} & =c_{t h} \kappa_{r}^{F_{r}}+\kappa_{z}^{F_{r}}=E A \frac{c^{2}}{s}\left[c_{t h}+s^{2}\left(1-c_{t h}\right)\right] \\
& +\frac{E I}{a^{2}} \frac{c^{4}}{s}\left(1+s^{2}\right)\left[c_{t h}+s^{2}\left(c_{t h}-1\right)\right]+\frac{G J}{a^{2}} s^{3} c^{2}\left[1+s^{2}\left(c_{t h}-1\right)\right]
\end{aligned}
$$


Eq. 20 yields for the case of equal radial and axial thermal strains $\left(c_{t h}=1\right.$, $\Delta T_{r}=\Delta T_{z}$ ), the following homogeneous $(h)$ thermal loading stiffness expressions:

$$
\begin{aligned}
\kappa_{\epsilon_{t h}}^{F_{z} h} & =\kappa_{r}^{F_{z}}+\kappa_{z}^{F_{z}}=E A s-\frac{E I}{a^{2}} s c^{4}+\frac{G J}{a^{2}} c^{4} s \\
\kappa_{\epsilon_{t h}}^{M_{z} h} & =\kappa_{r}^{M_{z}}+\kappa_{z}^{M_{z}}=E A a c+\frac{E I}{a} c^{3}\left(1+s^{2}\right)+\frac{G J}{a} s^{4} c \\
\kappa_{\epsilon_{t h}}^{F_{r} h} & =\kappa_{r}^{F_{r}}+\kappa_{z}^{F_{r}}=E A \frac{c^{2}}{s}+\frac{E I}{a^{2}} \frac{c^{4}}{s}\left(1+s^{2}\right)+\frac{G J}{a^{2}} s^{3} c^{2}
\end{aligned}
$$

The thermal loading stiffness expressions of Eqs. 20 and 21 are further numerically validated upon dedicated, parametrically constructed finite element models, over a wide range of structural arrangements and for different thermal loading inputs. The results indicate a very good agreement between the analytically and numerically computed thermal structural response, as explicated in Appendix A.

\subsection{Single layer cable structure under axial and thermal loads}

In the current section, we make use of the previously obtained thermal loading stiffness analytical formulas, to analyze the effect of temperature changes on the loading state of axially strained helical construction. To that scope, we consider the case study of single layer cable structures with six helical wires wound around a central straight core (Fig. 2). Thereupon, we analyze two distinct geometric arrangements, the first with a helix angle $\theta_{1}=73.0^{\circ}$ and the second with an angle $\theta_{2}$ equal to $80.8^{\circ}$. Their material and geometric parameters are summarized in Table 1:

Table 1: Material and geometric specifications

\begin{tabular}{c||c|c|c|c|c}
\hline Layer & $E\left(K N / \mathrm{mm}^{2}\right)$ & $r(\mathrm{~mm})$ & $\nu(-)$ & $\theta\left(^{\circ}\right)$ & $\alpha_{t h}\left(\cdot 10^{-6} 1 / o\right)$ \\
\hline Core & 197.9 & 1.970 & 0.3 & 90.0 & 17.3 \\
Helical Layer & 197.9 & 1.865 & 0.3 & $73.0 \mid 80.8$ & 17.3 \\
\hline
\end{tabular}

We consider that at the reference temperature state $(\Delta T=0)$, an axial strain of $\epsilon_{z}=0.01$ applies to both structural arrangements of Table 1. We analytically compute the mechanical force and moment resultants $\left[F_{z} M_{z} F_{r}\right]_{m}^{T}$ as a linear combination of the contributions arising from the helical constituents (Eq. B.2) and of the straight core.

Table 2: Cable loading state for a mechanical strain $\epsilon_{z}=0.01$ and $\Delta T=0$

\begin{tabular}{c||c|c|c}
\hline Layer angle $\theta\left(^{o}\right)$ & $F_{z}^{m}(k N)$ & $M_{z}^{m}(k N m m)$ & $F_{r}^{m}(k N)$ \\
\hline$\theta_{1}$ & 137.60 & 133.0 & 10.60 \\
$\theta_{2}$ & 148.93 & 77.5 & 3.27 \\
\hline
\end{tabular}


We subsequently apply homogeneous temperature changes of $\Delta T= \pm 10^{\circ}$ and $\Delta T= \pm 40^{\circ}$, for which we compute the variations $\Delta(\cdot):=\left((\cdot)_{\epsilon_{t h}}+\epsilon_{z}-(\cdot)_{\epsilon_{z}}\right) /(\cdot)_{\epsilon_{z}}$ (Fig. 2) of the effective $Q o I$ of Eq. 5 (left-hand side) acting on the structures of Table 1. In the variation expression, the $(\cdot)_{\epsilon_{z}}$ term represents the reference temperature state where no thermal loads apply so that the mechanical part $m$ of Eq. 5 equals to the effective, while the $(\cdot)_{\epsilon_{t h}+\epsilon_{z}}$ term for the state after the thermal load application where the thermal th needs to be subtracted from from the mechanical part $m$ for the effective forces and moments of Eq. 5 to be computed.

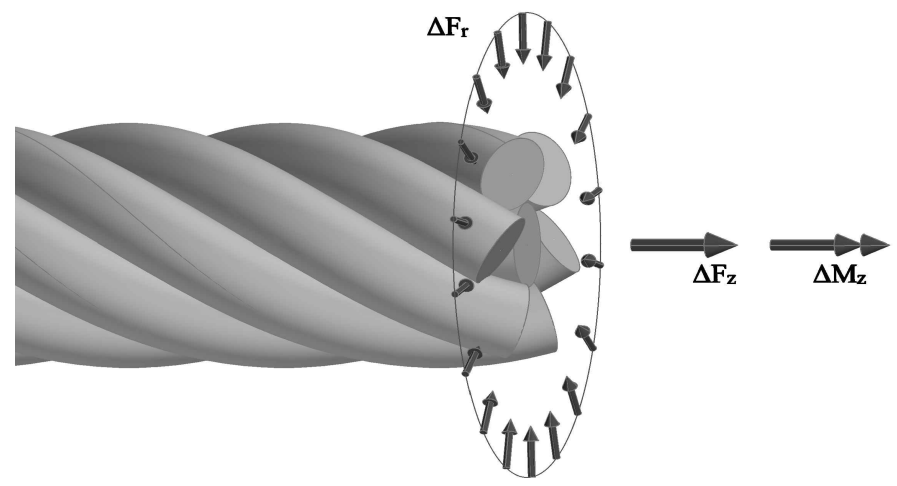

Figure 2: Single layer helical assembly

In Table 3, we summarize the thermal axial forces $F_{z}^{\epsilon_{t h}}(k N)$, moments $M_{z}^{\epsilon_{t h}}$ $(k N m m)$ and radial forces $F_{r}^{\epsilon_{t h}}(k N)$ induced in absolute values for the entire structure $\left(3^{r d}, 5^{\text {th }}\right.$ and $7^{\text {th }}$ column) along with the variation of the effective $Q o I$ of Eq. $5\left(4^{t h}, 6^{t h}\right.$ and $8^{t h}$ column). We note that the computed results do not account for the effect of possible thermal interactions between the helical constituents of the cable, computing the total cable thermal response as a simple linear superposition of the thermal responses of its constituents.

Table 3: Thermal forces and moments for temperature changes of 10 and 40 degrees and loading state variation upon the thermal load application

\begin{tabular}{c|c||c|c||c|c||c|c}
\hline & $\Delta T\left(^{\circ}\right)$ & $F_{z}^{\epsilon_{t h}}$ & $\Delta F_{z}$ & $M_{z}^{\epsilon_{t h}}$ & $\Delta M_{z}$ & $F_{r}^{\epsilon_{t h}}$ & $\Delta F_{r}$ \\
\hline \multirow{2}{*}{$\theta_{1}$} & \pm 10 & $\mp 2.5$ & $\mp 1.8$ & $\mp 2.5$ & $\mp 1.9$ & $\mp 0.21$ & $\mp 2.0$ \\
& \pm 40 & $\mp 10.0$ & $\mp 7.3$ & $\mp 10.0$ & $\mp 7.6$ & $\mp 0.84$ & $\mp 8.0$ \\
\hline \multirow{2}{*}{$\theta_{2}$} & \pm 10 & $\mp 2.6$ & $\mp 1.75$ & $\mp 1.4$ & $\mp 1.8$ & $\mp 0.06$ & $\mp 1.83$ \\
& \pm 40 & $\mp 10.4$ & $\mp 7.0$ & $\mp 5.6$ & $\mp 7.2$ & $\mp 0.24$ & $\mp 6.32$ \\
\hline
\end{tabular}

We note that a temperature increase reduces the forces and moments to which the structures are subjected, contrary to a temperature decrease that works as an additive tensioning mechanism (Table 3). For a temperature change 
of $10^{\circ}$, an axial force increase or decrease in the order of $2.5 \%$ takes place, a percentage that quadruples for a change of $40^{\circ}$. The different structural arrangements $\left(\theta_{1}, \theta_{2}\right)$ do not considerably influence the magnitude of the thermal axial forces $F_{z}^{\text {th }}$ created, their in between difference being smaller than $4 \%\left(3^{\text {rd }}\right.$ column of Table 3). However, they do have an impact on the thermally created torsional moment $M_{z}^{\epsilon_{t h}}$, as a quasi $50 \%$ lower thermal moment arises for the $\theta_{2}$ arrangement of $80.8^{\circ}$. Accordingly they substantially affect the magnitude of the radial thermal forces, which approximately quadruple upon a rather minor angle decrease of $\theta_{2}-\theta_{1}=7.8^{\circ}$ ( $7^{\text {th }}$ column of Table 3 ).

\section{Discussion and Conclusions}

The results of Section 3.2 suggest that the helical structure's loading state may be considerably influenced by rather low temperature variations $\Delta T$. Consequently, for the loading state to be accurately assessed, not only the mechanical loads but also the temperature variation limits under service conditions need to be accounted for. Furthermore, if a certain level of thermal insensitivity of the structural response is desired (variation of a certain QoI of Eq. 5 below a set bound), its material and geometric attributes need to be appropriately tuned, while a minimum mechanical load $(m)$ needs to be applied.

The magnitude of the thermally induced QoI in Eq. 5 depends not only on the structure's geometric and material attributes but also on the thermal loading input $\left(\epsilon_{r}^{t h}, \epsilon_{z}^{t h}\right)$, as the analytical closed-formed expressions of Eqs. 20 and 21 indicate. In order to obtain a graphical understanding of the previously mentioned dependencies, we depict in Fig. 3 both the homogeneous and the non-homogeneous stiffness formulas for $c_{t h}$ taking the values $0.5,1$ and 2 in a normalized form (denoted with $*$ ). We normalize with respect to $E A$ for the axial and radial force related terms and with respect to $E A a$ for the moment related terms. For the computations, we use a helix with an index $a / r=10$ $(r=0.1 m)$, material modulus $E=197.8 G P a$ and Poisson ratio $\nu=0.3$. 

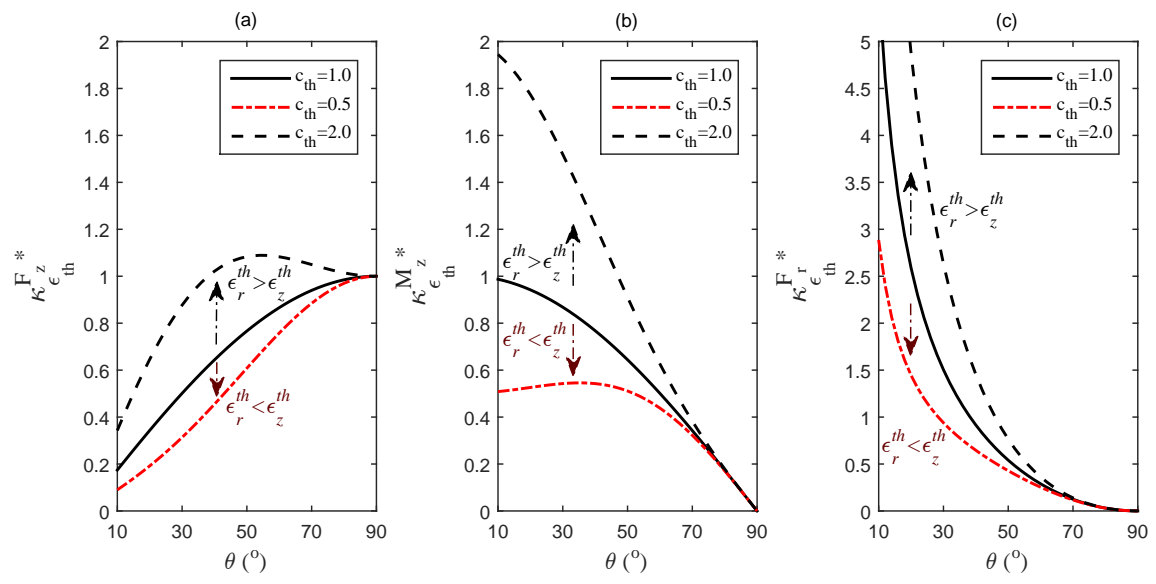

Figure 3: Helix thermal loading normalized axial (a) torsional (b) and radial (c) stiffness

The leftmost plot of Fig. 3 suggests that the helix thermal axial stiffness can be higher than the rod thermal axial stiffness $E A$ provided that a higher temperature change is exerted in the helix radial direction than along the helix axis $z\left(\epsilon_{r}^{t h} \succ \epsilon_{r}^{t h}\right)$. Nevertheless, the lower the ratio of the radial to the axial thermal strain applied is $\left(c_{t h}<1\right)$, the lower the resulting thermal axial force.

The helix angle $\theta$ regulates the significance of both the moment and radial force thermal stiffness related terms as plots (b) and (c) of Fig. 3 indicate. More specifically, helical arrangements of low helix angles $\theta$ develop considerably higher thermal moments and radial forces compared to arrangements of steep helix angles, with the absolute value to depend nonlinearly on the thermal loading input $\epsilon_{r}^{t h}$ and $\epsilon_{z}^{t h}$. Indicatively, for a radial thermal strain $\epsilon_{r}^{t h}$ that is two times the axial thermal strain $\left(c_{t h}=2\right)$, the thermal moment created is approximately three times the one induced for a radial strain that is half the axial strain $\left(c_{t h}=0.5\right)$, considering an angle $\theta$ of $30^{\circ}$.

We note that for high values of the helix angle and in particular for $\theta \succ 70^{\circ}$, the $\kappa_{\epsilon_{t h}}^{M_{z}{ }^{*}}$ and $\kappa_{\epsilon_{t h}}^{F_{r}{ }^{*}}$ terms are rather insensitive to $c_{t h}$, so that no significant differences arise between the homogeneous and non-homogeneous helix thermal loading mechanical responses. The argument can be verified upon a simple inspection of Eq. 20, where for steep helix angle values $\left(\theta \rightarrow 90^{\circ}\right)$, all $E I$ and $G J$ related contributions vanish $(\cos \theta \rightarrow 0)$, while the dependence on $c_{t h}$ for $E A$ drops $(\sin \theta \rightarrow 1)$.

Overall, we have provided thermal loading closed-form expressions that allow for the thermal structural response of the helical constructions to be assessed, taking explicitly into account the helix geometric and material attributes. Moreover, we have analyzed the effect of the thermal loading input (homogeneous and non-homogeneous) on the mechanical response, specifying the helix geometric parameter space within which, the incorporation of a possible non-homogeneity 
of the temperature field is an indispensable prerequisite of an accurate analysis. Finally, we have highlighted the formulas applicability in the realm of cable engineering, providing an insight into the effect of thermal loads on the loading state of single layer cables.

\section{Acknowledgements}

The work has been supported by the Swiss National Science Foundation under project No. 200021-137544-1.

\section{References}

[1] Steve Maas Shawn P. Reese and J. Weiss. Micromechanical models of helical superstructures in ligament and tendon fibers predict large Poisson's ratios. Journal of biomechanics, 43(7):1394-400, May 2010.

[2] M.H. Wei, K. Lin, L. Jin, and D.J. Zou. Nonlinear dynamics of a cablestayed beam driven by sub-harmonic and principal parametric resonance. International Journal of Mechanical Sciences, 110:78 - 93, 2016.

[3] Reginald Desroches Reza Mirzaeifar and Arash Yavari. A combined analytical, numerical, and experimental study of shape-memory-alloy helical springs. International Journal of Solids and Structures, 48(3-4):611-624, 2011.

[4] Moon, D.Y., G. Zi, J.H. Kim, S.J. Lee, G.Kim. On Strain Change of Prestressing Strand During Detensioning Procedures. Engineering Structures, 32:2570-2578, 2010.

[5] Raoof, M., T.J. Davies. Simple Determination of the Maximum Axial and Torsional Energy Dissipation in Large Diameter Spiral Strands. Computers and Structures, 84:676-689, 2006.

[6] M. Lepidi and V. Gattulli. Static and dynamic response of elastic suspended cables with thermal effects. International Journal of Solids and Structures, 49(9):1103-1116, 2012.

[7] T. Russell Gentry and M. Husain. Thermal Compatibility of Concrete and Composite Reinforcements, 1999.

[8] R. Byron Pipes and Pascal Hubert. Helical carbon nanotube arrays: thermal expansion. Composites Science and Technology, 63(11):1571-1579, 2003.

[9] Fernando Fraternali Ramathasan Thevamaran and Chiara Daraio. Multiscale Mass-Spring Model for High-Rate Compression of Vertically Aligned Carbon Nanotube Foams. Journal of Applied Mechanics, 81(12):121006, 2014. 
[10] F.H. Hruska. Tangential Forces in Wire Ropes. Wire and Wire Products, 28(5):455-460, 1953.

[11] S. Machida and A.J. Durelli. Response of a Strand to Axial and Torsional Displacements. Journal of Mechanical Engineering Science, 15:241-251, 1973.

[12] G.A. Costello. Theory of Wire Rope. Springer, New York, 1997.

[13] M.B.K. Moorthy S. Sathikh and M. Krishnan. A symmetric linear elastic model for helical wire strands under axisymmetric loads. The Journal of Strain Analysis for Engineering Design, 31(5):389-399, September 1996.

[14] N. Karathanasopoulos and G. Kress. Mechanical response of a helical body to axial, torsional and radial strain. International Journal of Mechanical Sciences, 9495:260 - 265, 2015.

[15] Papailiou, K.O. On the Bending Stiffness of Transmission Line Conductors. IEEE Transactions on Power Delivery, 12:1576-1588, 1997.

[16] W.S. Utting and N. Jones. The response of wire rope strands to axial tensile loadsPart I. Experimental results and theoretical predictions. International Journal of Mechanical Sciences, 29(9):605-619, January 1987.

[17] Utting, W.S. and N. Jones. The Response of Wire Rope Strands to Axial Tensile Loads-Part II: Comparison of Experimental Results and Theoretical Predictions. Int. J. Mech. Sci., 29(9):621-636, 1987.

[18] Messager, T., P. Cartraud. Homogenization of Helical Beam-Like Structures: Application to Single-Walled Carbon Nanotubes. Comput. Mech, 41:335-346, 2008.

[19] W.G. Jiang, M.S. Yao and J.M. Walton. A Concise Finite Element Model for Simple Straight Wire Rope Strand. International Journal of Mechanical Sciences, 41:143-161, 1999.

[20] E. Stanova, G. Fedorko, M. Fabian, and S. Kmet. Computer modelling of wire strands and ropes part II: Finite element-based applications. Advances in Engineering Software, 42(6):322-331, June 2011.

[21] W.G. Jiang and J.L. Henshall. A Novel Finite Element Model for Helical Springs. Finite Elements in Analysis and Design, 35:363-377, 2000.

[22] R. Wang and K. Ravi-Chandar. Mechanical Response of a Metallic Aortic StentPart I: Pressure-Diameter Relationship. Journal of Applied Mechanics, 71(5):697, 2004.

[23] Abdolhamid Meimandi-Parizi, Ahmad Oryan, and Ali Moshiri. Tendon Tissue Engineering and Its Role on Healing of the Experimentally Induced Large Tendon Defect Model in Rabbits: A Comprehensive In Vivo Study. PLoS ONE, 8(9), 2013. 
[24] Damien Durville Rachid Rahouadj Christian Geindreau Laurent Orgéas Cédric P. Laurent, Pierre Latil and Jean-François Ganghoffer. Mechanical behaviour of a fibrous scaffold for ligament tissue engineering: Finite elements analysis vs. X-ray tomography imaging. Journal of the Mechanical Behavior of Biomedical Materials, 40:222-233, 2014.

[25] G. Zhang and Y. Zhao. Mechanical characteristics of nanoscale springs. Journal of Applied Physics, 95(1):267, 2004.

[26] Ryan Sager Liming Dai Qiuhong Zhang, Jianwei Liu and Jeffery Baur. Hierarchical composites of carbon nanotubes on carbon fiber: Influence of growth condition on fiber tensile properties. Composites Science and Technology, 69(5):594-601, 2009.

[27] Yuan Liu, Zheng Yan, Qing Lin, Xuelin Guo, Mengdi Han, Kewang Nan, Keh-Chih Hwang, Yonggang Huang, Yihui Zhang, and John A. Rogers. Guided formation of 3d helical mesostructures by mechanical buckling: Analytical modeling and experimental validation. Advanced Functional Materials, Doi:10.1002/adfm.201505132, 2016.

[28] G. Mazzanti. Life Estimation of HVDC Cables Under the Time-Varying Electrothermal Stress Associated With Load Cycles. IEEE Transactions on Power Delivery, 30(2):931-939, 2015.

[29] Marco Giglio and a. Manes. Life prediction of a wire rope subjected to axial and bending loads. Engineering Failure Analysis, 12(4):549-568, 2005.

[30] W.K. Lee. An insight into wire rope geometry. International Journal of Solids and Structures, 28(4):471-490, 1991.

[31] A.S. Usmani, J M Rotter, S Lamont, A.M. Sanad, and M Gillie. Fundamental principles of structural behaviour under thermal effects. Fire Safety Journal, 36(8):721-744, 2001.

[32] H Ramsey. A theory of thin rods with application to helical constituent wires in cables. International Journal of Mechanical Sciences, 30(8):559$570,1988$. 


\section{Appendix A. Helix thermal loading finite element verification model}

A commercial, general purpose finite element program (Ansys) has been used to simulate the helix structural response to thermal loads. For the verification, different helix geometric configurations have been constructed, each of them generated by simulating a complete helix period $2 \pi$, discretized along the helix length by a mesh of 100 standard beam elements. Fig. A.4 below presents a model snapshot for a helix angle of $45^{\circ}$ and $a / r=20$.

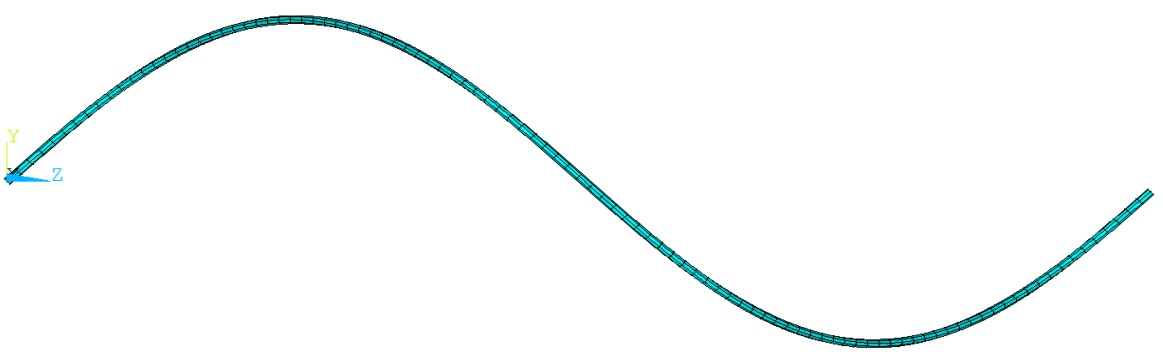

Figure A.4: Ansys helix model

For the comparisons, a case of homogeneous $\left(c_{t h}=1\right)$ and two cases of inhomogeneous thermal loads have been considered with $c_{t h}=0.5$ and $c_{t h}=2$ accordingly. For each thermal loading case, helical bodies with circular cross sections of radius $r$ have been build with helix index values $a_{i} / r$ as low as 10 and up to $300(r=1 \mathrm{~cm})$, upon an increment of 10 . For each helix index value $a / r$, a total of 17 different helix angle values $\theta$ have been simulated, from $5^{o}$ up to $90^{\circ}$ upon a $5^{\circ}$ increment. Table A. 4 summarizes the parametric space considered:

Table A.4: Helix thermal loading validation parametric space

\begin{tabular}{c||c}
$\theta_{i}\left(^{o}\right)$ & $5: 5: 90$ \\
$\alpha_{i} / r(-)$ & $10: 10: 300$ \\
\hline
\end{tabular}

For the analysis, a linear elastic modulus $E=197.9 \mathrm{GPa}$ has been used with a Poisson ratio $\nu=0.3$ and a thermal coefficient $\alpha_{t h}=17 \cdot 10^{-6}(1 / o)$. In the case of homogeneous thermal loading $\left(c_{t h}=1\right)$, a temperature difference $\Delta T=10^{\circ}$ has been applied to all models, while the axial, torsional and radial deformation of all nodes along the helix have been constrained.

For the case of inhomogeneous thermal loading, the force and moment resultants of Eq. 5 have been retrieved upon the superposition of the radial and axial thermal strain application force and moment resultants $\left(\Delta T_{r}=10^{\circ}, c_{t h}=0.5\right.$ and $c_{t h}=2$ ). Upon the radial thermal strain application, the torsional and axial deformation of all nodes along the helix period were constrained, while 
upon the axial thermal strain application, the radial and torsional deformation of all nodes were suppressed, along with the axial deformation of the helix start $(\varphi=0)$ to avoid rigid body motions.

The comparisons between the numerical results and the analytical results computed through Eqs. 20 and 21 revealed differences lower than 1\% in the entire parametric space defined in Table A.4 and for all QoI of Eq. 5. In Fig. A.5, a case study comparison between the analytically and numerically obtained forces and moments of Eq. 5 is presented for $a / r=10$ and $c_{t h}=1$ and for all helix angle values $\theta$ defined in Table A.4:
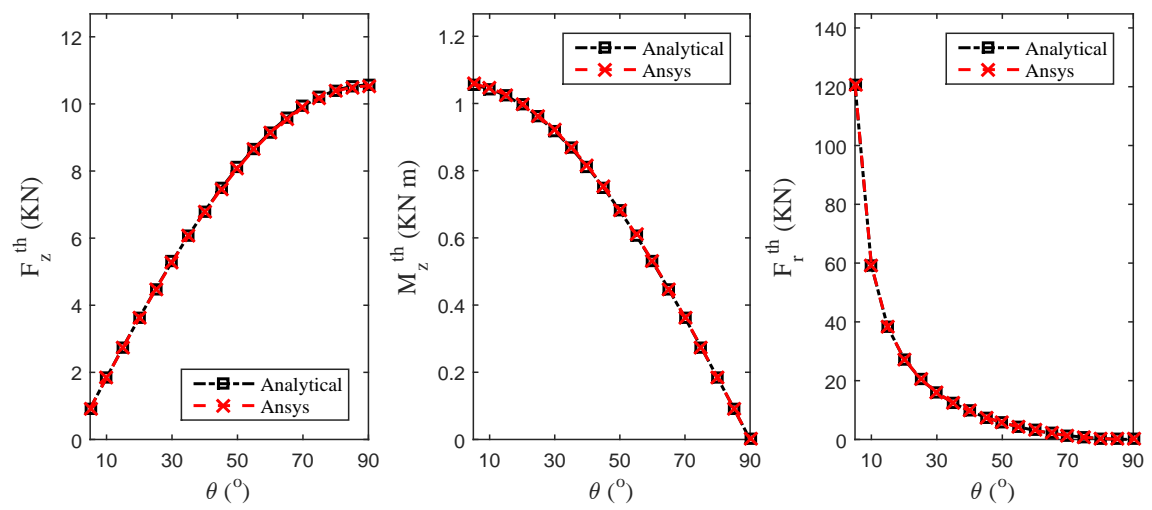

Figure A.5: A case study comparison of the numerically and analytically obtained thermal structural response

\section{Appendix B. Analytical formulas of the structural response of heli- cal constructions to mechanical strains}

The helix linear structural response to mechanical strains, namely to axial $\epsilon_{z}\left(\epsilon_{z}=\delta h / h\right)$, torsional $\omega^{\prime}\left(\omega^{\prime}=\delta \varphi / h\right)$ and radial strains $\epsilon_{r}\left(\epsilon_{r}=\delta a / a\right)$ is characterized for the mechanical force and moment $Q o I$ of Eq. 5 by means of a $3 \times 3$ stiffness matrix, defined as follows:

$$
\left[\begin{array}{c}
F_{z} \\
M_{z} \\
F_{r}
\end{array}\right]_{m}=\left[\begin{array}{lll}
\kappa_{\epsilon_{z} \epsilon_{z}} & \kappa_{\epsilon_{z} \omega^{\prime}} & \kappa_{\epsilon_{z} \epsilon_{r}} \\
\kappa_{\omega^{\prime} \epsilon_{z}} & \kappa_{\omega^{\prime} \omega^{\prime}} & \kappa_{\omega^{\prime} \epsilon_{r}} \\
\kappa_{\epsilon_{r} \epsilon_{z}} & \kappa_{\epsilon_{r} \omega^{\prime}} & \kappa_{\epsilon_{r} \epsilon_{r}}
\end{array}\right]\left[\begin{array}{c}
\epsilon_{z} \\
\omega^{\prime} \\
\epsilon_{r}
\end{array}\right]
$$


The stiffness term components are given as a function of the material and geometric parameters defined in Section 2, as follows [14]:

$$
\begin{aligned}
& \kappa_{\epsilon_{z} \epsilon_{z}}=E A s^{3}+\frac{E I}{a^{2}} s^{3} c^{4}+\frac{G J}{a^{2}} s c^{6} \\
& \kappa_{\omega^{\prime} \omega^{\prime}}=E A a^{2} c^{2} s+E I c^{2} s\left(1+s^{2}\right)^{2}+G J s^{7} \\
& \kappa_{\epsilon_{r} \epsilon_{r}}=E A \frac{c^{4}}{s}+\frac{E I}{a^{2}} \frac{c^{4}}{s}\left(1+s^{2}\right)^{2}+\frac{G J}{a^{2}} s^{5} c^{2} \\
& \kappa_{\omega^{\prime} \epsilon_{z}}=\kappa_{\epsilon_{z} \omega^{\prime}}=E A a c s^{2}-\frac{E I}{a} s^{2} c^{3}\left(1+s^{2}\right)+\frac{G J}{a} s^{4} c^{3} \\
& \kappa_{\epsilon_{r} \epsilon_{z}}=\kappa_{\epsilon_{z} \epsilon_{r}}=E A c^{2} s-\frac{E I}{a^{2}} s c^{4}\left(1+s^{2}\right)+\frac{G J}{a^{2}} s^{3} c^{4} \\
& \kappa_{\omega^{\prime} \epsilon_{r}}=\kappa_{\epsilon_{r} \omega^{\prime}}=E A a c^{3}+\frac{E I}{a} c^{3}\left(1+s^{2}\right)^{2}+\frac{G J}{a} s^{6} c
\end{aligned}
$$

